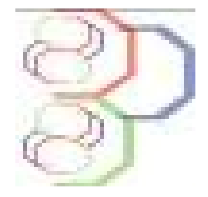

ISSN 1997-5902

\title{
Dynamique des populations du foreur des tiges du cacaoyer, Eulophonotus myrmeleon Felder (Lépidoptère : Cossidae) dans la région du Haut-Sassandra en Côte d'ivoire
}

\author{
N'Guessan ${ }^{1}$ A.H. ${ }^{*}$, N'Guessan ${ }^{1}$ K.F., Kouassi2 K.P., Kouamé ${ }^{12}$ N.N. ${ }^{*}$, N'Guessan ${ }^{1}$ P.W. \\ ${ }^{1}$ CNRA, Station de Recherche de Divo, BP 808 Divo, Côte d'Ivoire ; \\ 2 Université de Cocody, UFR Biosciences, Labo. Zoologie et Bio. Animal, 22 BP 582 Abidjan 22 ; \\ Auteur correspondant e-mail : hauverset@yahoo.fr ; Tel: (225) 06565476
}

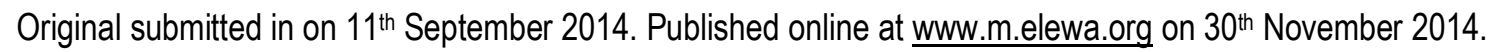

http://dx.doi.org/10.4314/iab.v83i1.11

\section{RESUME}

Objectif : Le foreur de tiges du cacaoyer, Eulophonotus myrmeleon Felder cause aujourd'hui d'énormes dégâts dans les cacaoyères ivoiriennes. La présente étude vise à déterminer les périodes de fortes attaques de ce déprédateur dans la région du Haut-Sassandra, la deuxième plus grande région de production de cacao en Côte d'ivoire.

Méthodologie et résultats : L'étude a été réalisée de 2009 à 2013 dans les plantations villageoises de la région du Haut-Sassandra en Côte d'Ivoire. Les variations des taux d'attaques d'Eulophonotus myrmeleon ont été évaluées par dénombrement mensuel des trous récents dans six cacaoyères attaquées, choisies dans trois sous-préfectures, à raison de 2 par sous-préfecture. Les données de pluviométrie et de température ont été également collectées durant cette étude. Les résultats obtenus ont montré qu'il y a deux périodes de fortes attaques de ce déprédateur au cours de l'année dans la région du Haut-Sassandra. Ces périodes se situent de Janvier à Avril avec un pic en Février et de Juin à Novembre avec un pic en Août. Pendant ces périodes, la population des larves est très élevée dans les cacaoyères, indiquant une importante activité larvaire dans les cacaoyers. Les résultats ont aussi montré que le niveau d'attaques a augmenté de 2009 à 2013, indiquant que cet insecte est devenu une préoccupation majeure pour les producteurs de cette région. La pluviométrie semble jouer un rôle important dans la pullulation de cet insecte car les périodes de fortes attaques ont été observées après les périodes de fortes précipitations. De même, les baisses de température semblent favoriser un accroissement des attaques d'Eulophonotus myrmeleon.

Conclusion et applications : Les résultats obtenus peuvent être utiles pour faire des recommandations de lutte contre les larves et les adultes. En effet, les périodes de fortes attaques au cours desquelles le niveau de population des larves est élevé dans les cacaoyères, et les périodes d'émergence des adultes pourraient être prises en compte dans les interventions phytosanitaires, notamment une application rationnelle d'insecticide. Mots clés : Cacaoyer, foreur de tiges, Eulophonotus myrmeleon, dynamique des populations, Côte d'Ivoire. 


\title{
N'Guessan et al. J. Appl. Biosci. 2014 Dynamique des populations du foreur des tiges du cacaoyer,
} Eulophonotus myrmeleon Felder (Lépidoptère : Cossidae) dans la région du Haut-Sassandra en Côte d'ivoire

Population dynamic of the cocoa stem borer, Eulophonotus myrmeleon Felder (Lepidoptera: Cossidae) in the Haut-Sassandra region of Côte d'Ivoire

\begin{abstract}
Objective: The cocoa stem borer, Eulophonotus myrmeleon Felder causes today serious damage in cocoa farm in Côte d'Ivoire. This study was conducted to determine the outbreak periods of this new lepidopteran pest in the Haut-Sassandra region, the second largest cocoa producing area in Côte d'Ivoire.

Methodology and Results: The study was conducted from 2009 to 2013 in farmers' cocoa farms in the HautSassandra region in Côte d'Ivoire. Seasonal variation of the rate of Eulophonotus myrmeleon attacks were assessed by counting recent holes in six cocoa farms chosen in 3 counties with 2 farms in each county. Rainfall data were also collected during the same period. The results showed that there are two periods of severe attacks of $E$ myrmeleon in the Haut-Sassandra region. These attacks occur from January to April with a peak in February and from June to November with a peak in August. During these outbreak periods, the larval population level is high in the cocoa farms, indicating intense larval activities in the farms. The results also showed that the level of attack increased from 2009 to 2013, indicating that this insect has become a serious problem for the farmers in this region. Rainfall seems to play a significant role in the breeding and population rise of these insects as periods of heavy attacks were observed after periods of rainfalls. Similarly, the lower temperatures seemed to favor an increase in Eulophonotus myrmeleon attack.

Conclusion and applications: The results obtained in this study could be very useful for recommending control measures against larvae or adults. Indeed, periods of heavy attacks and adult emergence could be taken into account for rational insecticide application against this pest.
\end{abstract}

Keywords: Cocoa tree, stem borer, Eulophonotus myrmeleon, population dynamic, Côte d'Ivoire.

\section{INTRODUCTION}

Depuis 1977, la Côte d'Ivoire est le premier producteur mondial de cacao. Aujourd'hui, la production ivoirienne qui s'est accrue progressivement, atteint 1,476 millions de tonnes et représente $40 \%$ de la production mondiale (ICCO, 2012). La cacaoculture est pratiquée sur une superficie de 2 millions d'hectares environ, dans la partie sud du pays et concerne une population agricole de plus de 1000000 de planteurs (ICCO, 2008). Le cacao génère plus de $30 \%$ des recettes d'exportation et participe à plus de $15 \%$ au Produit Intérieur Brut. II fait vivre 4 millions de personnes, soit $25 \%$ de la population. Cependant, en dépit de ces performances assez remarquables, la cacaoculture ivoirienne est confrontée à de nombreuses contraintes qui menacent la durabilité de la culture. En effet, il existe un vaste cortège d'insectes nuisibles du cacaoyer dont les plus dommageables sont les mirides, la punaise verte, les chenilles défoliatrices et les foreurs de tiges. Les foreurs des tiges étaient considérés autrefois comme déprédateurs mineurs du cacaoyer. Les plus connus étaient Phosphorus virescens, Phosphorus gabonator, Tragocephala castnia and Apate monachus. Ceux-ci se trouvaient aussi sur d'autres arbres tels que le colatier et le caféier (Daramola, 1978; Ojo, 1981, Ndubuaku, 1989; Okolena, 1989). Cependant, depuis les années 1990, le foreur de tiges Eulophonotus myrmeleon est devenu préoccupant pour les producteurs. Cet insecte cause des dégâts de plus en plus importants dans les cacaoyères ivoiriennes. Ce qui suscite de nombreuses plaintes de la part des producteurs. Face à cette situation, plusieurs études ont été menées par la recherche notamment sur les dégâts causés par cet insecte (N'Guessan, 2007). En matière de lutte contre ces déprédateurs, très peu de travaux ont été réalisés (Anikwe, 2010). Certains producteurs utilisent la lutte mécanique consistant à boucher les trous de l'insecte pour limiter les dégâts ; toutefois, cette méthode s'avère inefficace (Anikwe, 2010). Les pratiques qui consistaient à injecter à l'intérieur des galeries du pétrole, de l'essence, du chloroforme ou du paradichlorobenzène pour lutter contre ces insectes se sont avérées également inefficaces et difficiles 


\section{N'Guessan et al. J. Appl. Biosci. 2014 Dynamique des populations du foreur des tiges du cacaoyer,}

Eulophonotus myrmeleon Felder (Lépidoptère : Cossidae) dans la région du Haut-Sassandra en Côte d'ivoire

d'application (Vos et al. 2003). Par ailleurs, dans le souci de proposer des mesures de lutte contre ce déprédateur, des études ont été entreprises pour déterminer les périodes de fortes pullulation dans les principales régions de production du pays

\section{MATERIEL ET METHODES}

Site d'étude : Les études ont été réalisées en 2009-2013 en milieu paysan dans la région du Haut-Sassandra. Cette région qui est située dans la partie Centre-Ouest du pays, constitue la 2 ème principale région de production en Côte d'lvoire, avec plus de $12 \%$ de la production nationale chaque année. Trois localités représentatives de la zone de production de cacao ont été choisies (Daloa, Issia et Zoukougbeu) pour l'échantillonnage de l'insecte.

Méthode d'échantillonnage: Dans chaque localité choisie, 2 sites d'échantillonnage constitués par des cacaoyères d'au moins 1 ha, attaquées par le foreur des tiges, E. myrmeleon, ont été identifiés, soit au total six cacaoyères. II s'agit des plantations de Messieurs Touhapli Kanon Etienne (Daloa), Kabré Elie (Daloa), Goudjé Fidèle (Zoukougbeu), Ouili Michel (Zoukougbeu), Daleba G. Paul (Issia) et Biazo Salomon (Issia). Dans chaque cacaoyère 3 blocs de 100 cacaoyers ont été délimités et les arbres ont été numérotés de 1 à 100 à l'aide de la peinture à huile de couleur rouge. Ceci fait 300 cacaoyers par parcelle élémentaire, soit un total de 1800 cacaoyers observés dans les cacaoyères tous les mois. Toutes les cacaoyères choisies étaient restées sans traitement insecticides au cours de l'étude.

Observations : Dans chaque parcelle élémentaire, les cacaoyers numérotés ont été inspectés tous les mois depuis le démarrage de l'étude, sachant, à partir d'étude antérieures (Entwistle, 1972), que les larves

\section{RESULTATS}

Après cinq années d'observation sur les attaques d'Eulophonotus myrmeleon dans les cacaoyères de la région du Haut-Sassandra, l'analyse de variance a révélé des différences significatives $(P<0,05)$ entre les 3 localités échantillonnées au regard du taux d'attaques et de la population des larves d'Eulophonotus myrmeleon, représenté par le nombre de trous récents dans les cacaoyères (Tableau 1). La population des larves et le taux de cacaoyers attaqués se sont avérés légèrement plus élevés dans les cacaoyères de la sous-préfecture d'Issia que dans celles de Zoukougbeu et Daloa (Tableau
(N'Guessan et al., 2010). C'est dans ce contexte que la présente étude a été réalisée dans la région $d u$ Haut-Sassandra, l'une des principales régions de production de cacao en Côte d'Ivoire.

d'Eulophonotus myrmeleon peuvent creuser des galeries dans le cacaoyer pendant 3 mois. Les orifices récents ont été dénombrés jusqu'à une hauteur de $2 \mathrm{~m}$ (à portée de main) sur les cacaoyers marqués. Ces orifices récents étaient reconnaissables par la présence de déchets rougeâtres qui s'échappaient des trous sur les troncs. La présence de déchets montre qu'une larve vivante et active creuse des galeries dans le tronc. Tous les orifices récents ont été systématiquement dénombrés sans considérer le comptage précédent. Ainsi, le nombre d'orifices dénombrés à chaque passage était un nombre cumulé. Les données de précipitations ont été collectées 2 fois par jour (matin et après-midi) sur les stations météorologiques de l'Agence Nationale d'Appui au Développement Rural (ANADER) dans les localités concernées par l'étude.

Analyse des données: Les données collectées, notamment le nombre de trous récents par arbre et le nombre d'arbres attaqués ont été soumises à une analyse de variance à l'aide de la procédure GLM (General Linear Model) du logiciel SAS 9.2. La comparaison des moyennes a été réalisée par le test de Waller Duncan K-ration T-test, au seuil de 5.\%. Par ailleurs, le nombre moyen d'orifice par arbre a été déterminé et représenté graphiquement pour évaluer les variations saisonnières des dégâts ou du niveau de population en fonction du régime pluviométrique et de la température.

1). Par ailleurs, l'analyse de variance a révélé des différences significatives $(P<0,05)$ entre les années d'échantillonnage au regard du taux d'attaques et de la population des larves d'Eulophonotus myrmeleon. La population des larves et le taux d'attaques ont varié d'une année à l'autre (Tableau 2). Le niveau de population des larves était plus faible en 2009 et 2010 comparativement à 2011, 2012 et 2013. De même, le taux d'attaques était relativement plus faible en 2009 et 2010 par rapport à 2011, 2012 et 2013 (Tableau 2). 
N'Guessan et al. J. Appl. Biosci. 2014 Dynamique des populations du foreur des tiges du cacaoyer, Eulophonotus myrmeleon Felder (Lépidoptère : Cossidae) dans la région du Haut-Sassandra en Côte d'ivoire

Tableau 1: Nombre moyen de trous récents et taux d'attaques d'Eulophonotus myrmeleon dans les cacaoyères de trois sous-préfectures de la région du Haut-Sassandra en Côte d'Ivoire.

\begin{tabular}{lcc}
\hline Sous-préfectures échantillonnées & $\begin{array}{c}\text { Nombre moyen de trous } \\
\text { récents par arbre }\end{array}$ & Taux d'attaques (\%) \\
\hline Issia & $8,86 \pm 1,28 \mathrm{a}$ & $5,75 \pm 0,63 \mathrm{a}$ \\
Zoukougbeu & $4,98 \pm 0,74 \mathrm{~b}$ & $3,35 \pm 0,33 \mathrm{~b}$ \\
Daloa & $3,38 \pm 0,40 \mathrm{~b}$ & $2,56 \pm 0,27 \mathrm{~b}$ \\
\hline Les moyennes suivies d'une même lettre dans une colonne sont statistiquementidentiques au seuil de $5 \%$ (Waller Duncan K-ration
\end{tabular}

Tableau 2: Nombre moyen de trous récents et taux d'attaques d'Eulophonotus myrmeleon pour 5 années consécutives dans les cacaoyères de la région du Haut-Sassandra en Côte d'Ivoire.

\begin{tabular}{lcc}
\hline Années & $\begin{array}{c}\text { Nombre moyen de trous récents par } \\
\text { arbre }\end{array}$ & Taux d'attaques (\%) \\
\hline 2013 & $13,36 \pm 2,03 \mathrm{a}$ & $7,81 \pm 0,93 \mathrm{a}$ \\
2012 & $6,19 \pm 1,02 \mathrm{~b}$ & $4,12 \pm 0,46 \mathrm{~b}$ \\
2011 & $5,34 \pm 0,78 \mathrm{~b}$ & $3,91 \pm 0,54 \mathrm{~b}$ \\
2010 & $1,44 \pm 0,23 \mathrm{c}$ & $1,39 \pm 0,18 \mathrm{c}$ \\
2009 & $2,36 \pm 0,37 \mathrm{c}$ & $2,20 \pm 0,30 \mathrm{c}$ \\
\hline
\end{tabular}

Les moyennes suivies d'une même lettre dans une colonne sont statistiquement identiques au seuil de $5 \%$ (Waller Duncan K-ration T-test)

L'analyse des courbes de pullulations d'Eulophonotus myrmeleon des localités d'Issia, Daloa et Zoukougbeu permet de distinguer deux périodes de fortes attaques au cours de l'année (Figure 1). Dans la localité d'Issia, les périodes de fortes attaques s'étalent de Novembre à Mars avec un pic en Janvier ou Février, et de Juin à Novembre avec un pic en Août. Dans les localités de
Zoukougbeu, les périodes de fortes attaques s'étalent de Décembre à Mai avec un pic en Février et de Juin à Novembre avec un pic en Août. Dans les localités de Daloa, les fortes périodes d'attaque vont de Novembre à Mars avec un pic en Février et de Mai à Octobre avec un pic en Août.

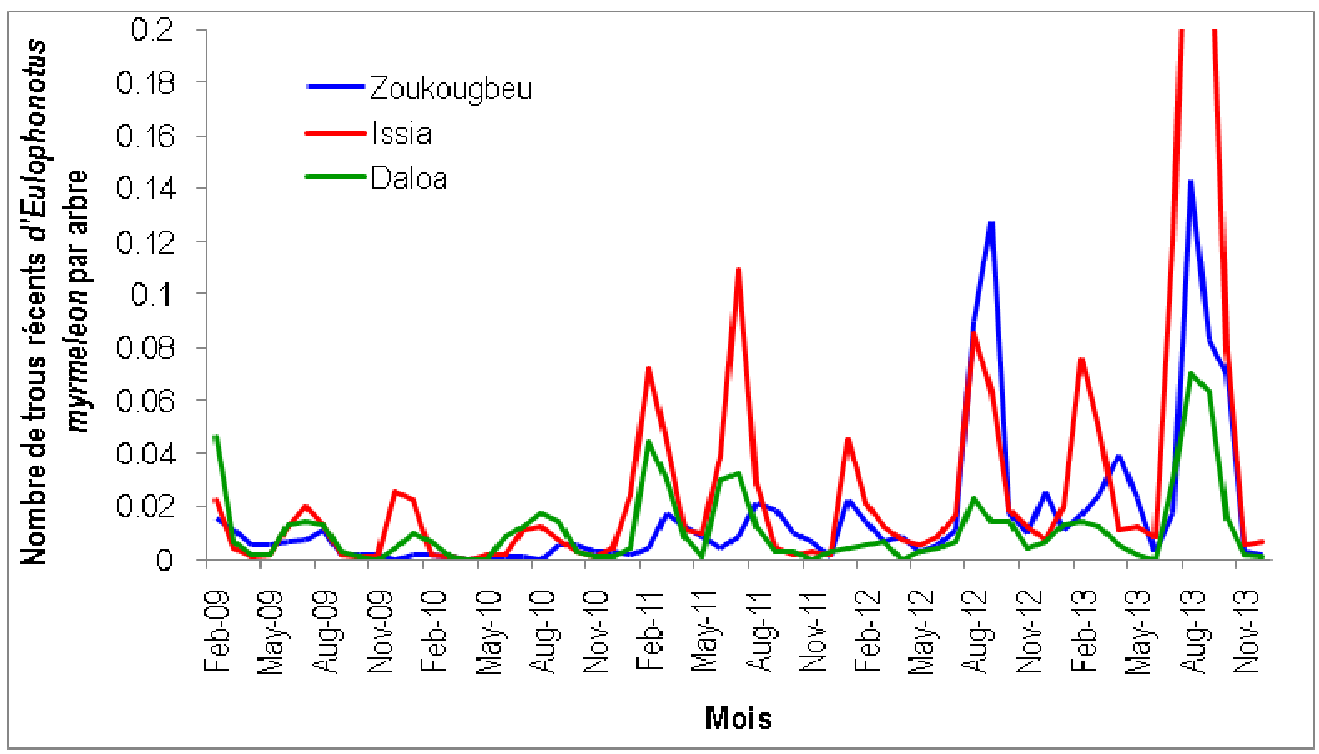

Figure 1. Variations saisonnières du nombre de trous récents d'Eulophonotus myrmeleon contenant des larves actives dans trois sous-préfectures de la région du Haut-Sassandra en Côte d'Ivoire. 
La courbe moyenne de pullulation d'Eulophonotus myrmeleon dans la région du Haut-Sassandra (Figures 2 et 3) confirme deux périodes de fortes attaques. Ces périodes s'étalent de Janvier à Avril avec un pic en Février et de Juin à Octobre avec un pic en Août. Ces périodes correspondent aux moments où le niveau de population des larves est élevé dans les cacaoyères. La première phase d'attaques commence dans le mois de Janvier, augmente progressivement pour atteindre un pic en Février avant de diminuer d'intensité jusqu'au mois d'Avril. Ensuite, une deuxième phase commence au mois de Juin avec un pic en Août puis décroît jusqu'en Novembre. Au-delà de ces deux périodes, les attaques étaient relativement faibles, voire négligeables au cours des périodes d'Octobre à Novembre et Mars à Mai chaque année. Au cours des périodes de fortes attaques, les larves creusent des galeries dans les troncs et les tiges des cacaoyers (Figure 4). Les premiers adultes sont visibles dans les parcelles à partir du mois d'Avril puis par la suite dans le mois de Septembre, c'est-à-dire trois mois après le début des attaques en Janvier et Juin. Les données pluviométriques ont révélé qu'au cours de notre étude, les niveaux de précipitations étaient élevé dans tous les mois sauf en décembre et Janvier, et d'une moindre importance en Novembre (Figures 2 et 3 ). L'analyse de l'évolution des attaques d'Eulophonotus myrmeleon en fonction de la pluviométrie révèle que les périodes de fortes attaques apparaissent après les périodes de fortes précipitations, ou pendant les périodes pluvieuses (Figure 3). De même, les attaques diminuent progressivement à partir des périodes sèches. Les températures moyennes enregistrées pendant les 5 années d'observation ont varié de $24^{\circ} \mathrm{C}$ à $27^{\circ} \mathrm{C}$. Les températures sont basses dans les mois de juillet, août et septembre. Les températures les plus élevées se situent en Février, Mars et Avril. La figure 6 montre que la plus grande période de forte pullulation d'Eulophonotus myrmeleon coïncide avec les températures les plus basses de l'année.

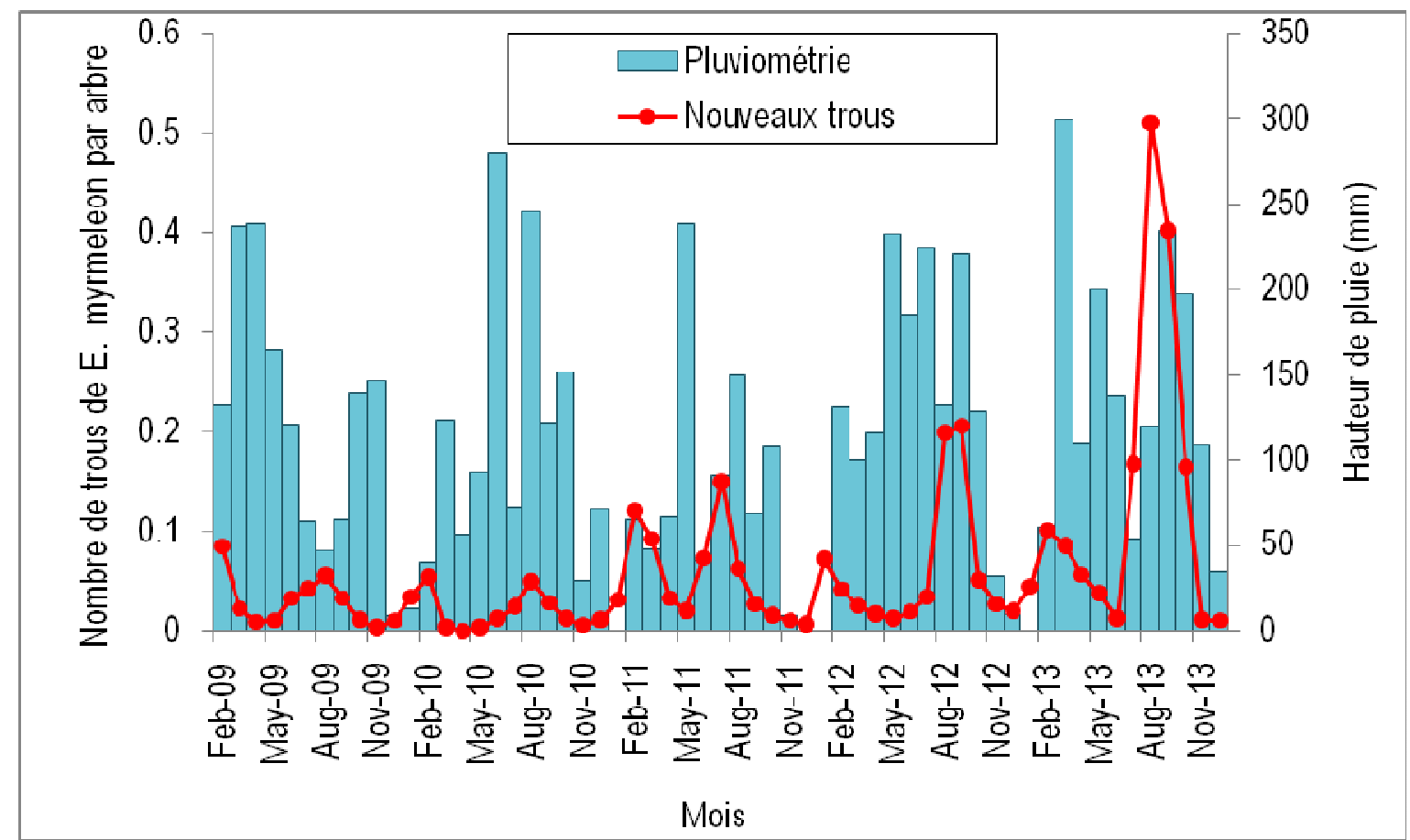

Figure 2: Variations saisonnières du nombre de trous récents d'Eulophonotus myrmeleon contenant des larves actives dans les cacaoyères du Haut-Sassandra en fonction de la pluviométrie 
N'Guessan et al. J. Appl. Biosci. 2014 Dynamique des populations du foreur des tiges du cacaoyer, Eulophonotus myrmeleon Felder (Lépidoptère : Cossidae) dans la région du Haut-Sassandra en Côte d'ivoire

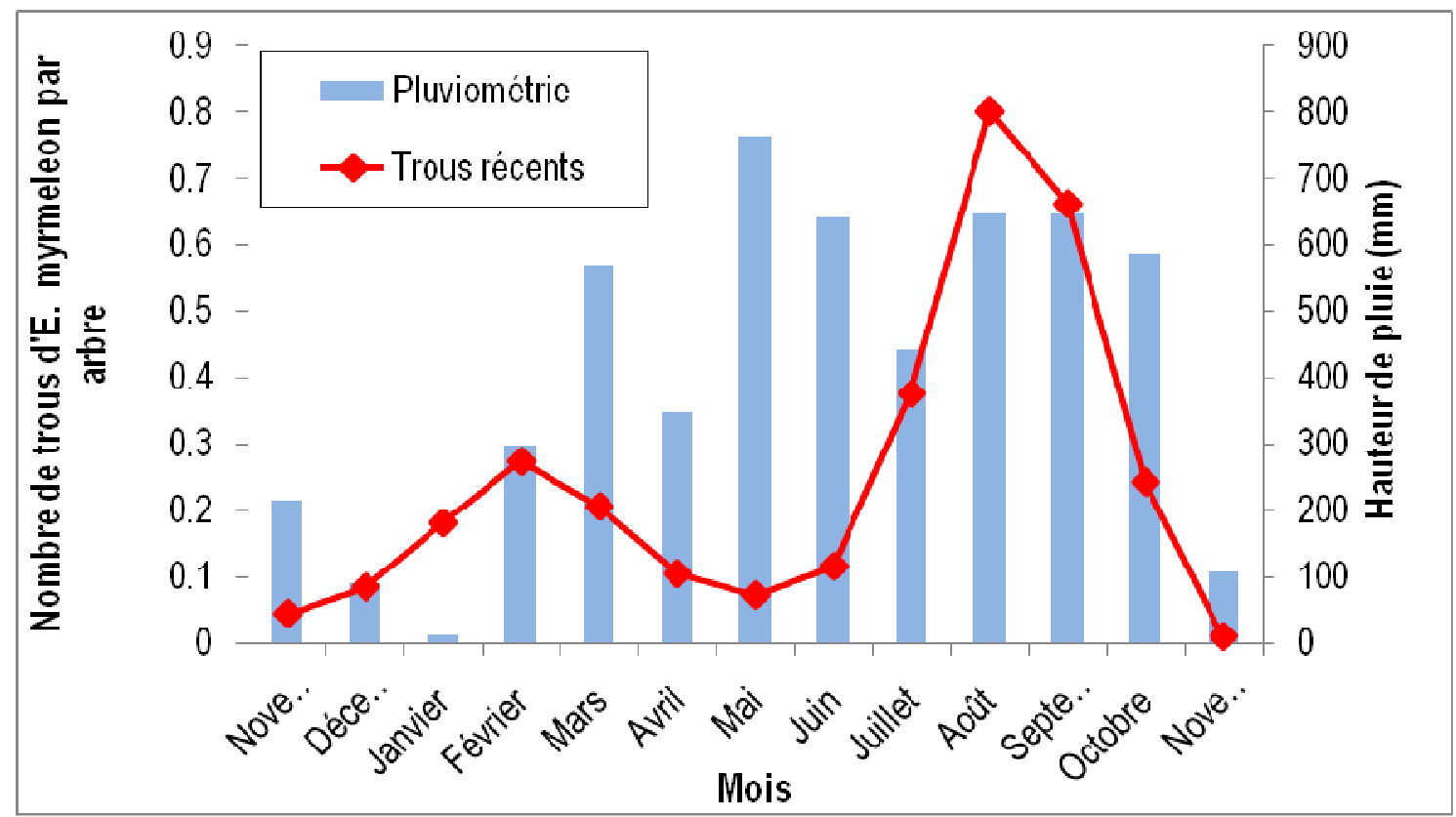

Figure 3: Variations mensuelles du nombre moyen de trous récents d'Eulophonotus myrmeleon contenant des larves actives dans les cacaoyères du Haut-Sassandra en fonction de la pluviométrie

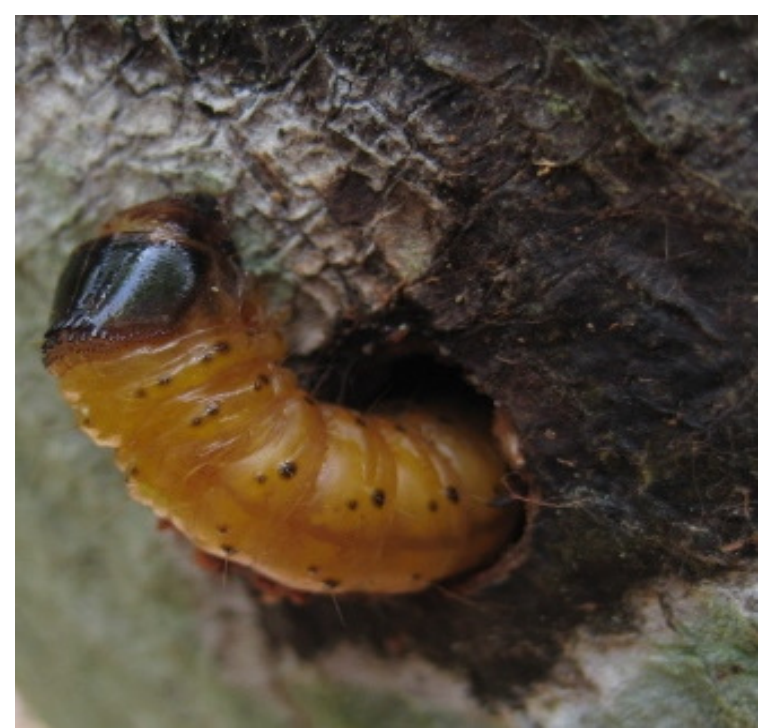

Figure 4: Larve d'Eulophonotus myrmeleon creusant une galerie dans le tronc d'un cacaoyer

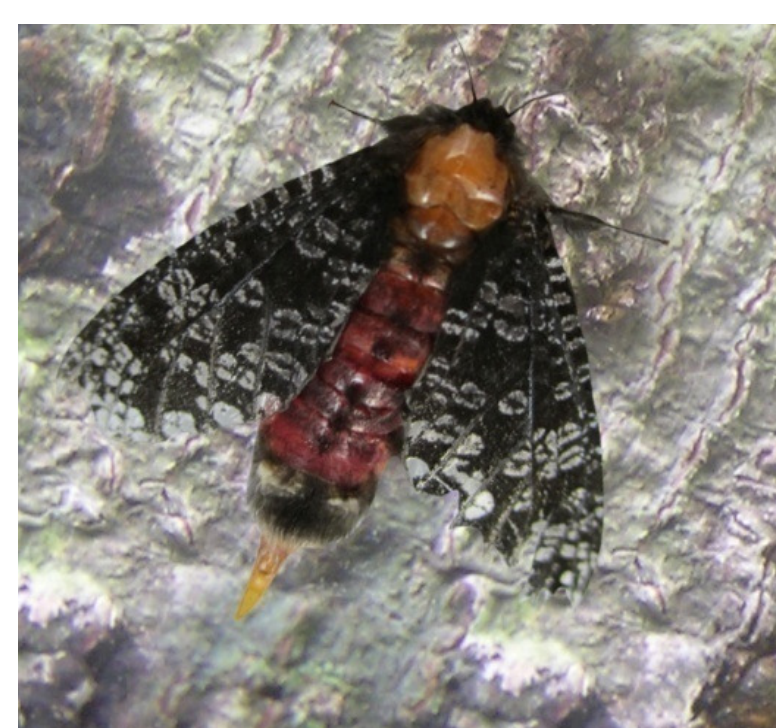

Figure 5: Adulte d'Eulophonotus myrmeleon 


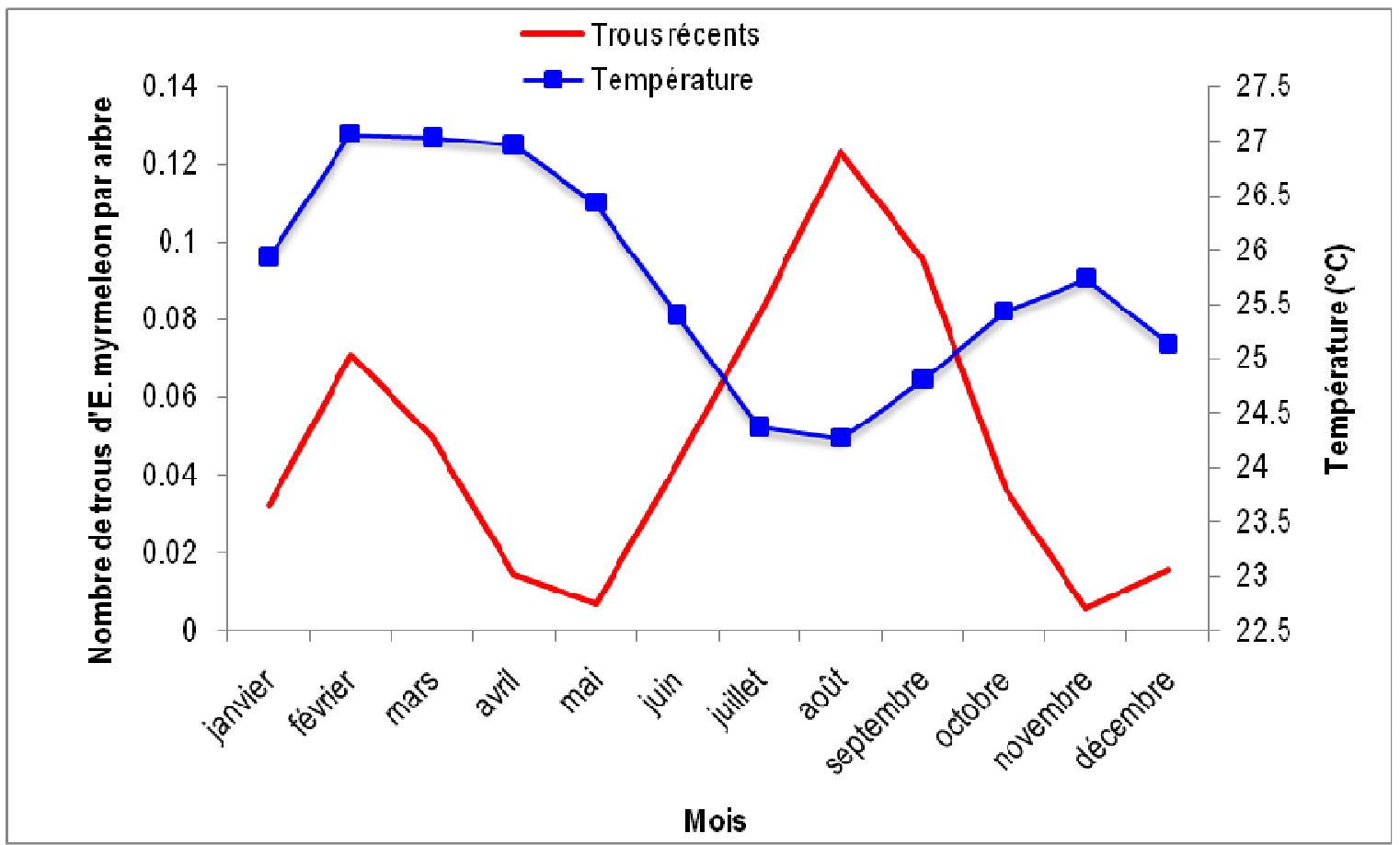

Figure 6 : Variations mensuelles du nombre moyen de trous récents d'Eulophonotus myrmeleon contenant des larves actives et de la température dans les cacaoyères du Haut-Sassandra.

\section{DISCUSSION}

L'étude de la dynamique des populations d'Eulophonotus myrmeleon dans la région du Haut-Sassandra révèle un accroissement des dégâts dans le temps avec des dégâts plus importants en 2013 qu'en 2009. Ces résultats suggèrent que d'Eulophonotus myrmeleon est devenu un déprédateur important dans cette région. Caplong et al. (1993) avaient indiqué que ce ravageur qui était mineur dans les cacaoyères de la Côte d'Ivoire dans le passé, était devenu préoccupant depuis les années 1990 avec des dégâts importants que dans la région Est du pays. Dans la même période, Wegbe et al. (1995) et Padi and Adu-Acheampong (2000) avaient noté une recrudescence d'Eulophonotus myrmeleon respectivement au Togo et au Ghana. La recrudescence de ce ravageur dans les cacaoyères des pays producteurs de l'Afrique de l'Ouest pourrait s'expliquer par une disparition progressive des plantes hôtes autres que le cacaoyer, en raison de la déforestation (Pallix et Comolet, 1996). En effet cet insecte était connu au Cameroun, en Côte d'Ivoire, au Ghana, au Nigeria, en Sierra Leone, au Togo, à Sao Tomé, en République Démocratique du Congo et en Afrique du Sud comme insecte qui s'attaque principalement aux espèces végétales de la famille Sterculiaceae (Entwistle, 1963, 1972). N'Guessan (2007) a indiqué qu'en Côte d'Ivoire, les attaques qui avaient débuté dans la région Est du pays, progressaient rapidement à l'Ouest. Nos résultats indiquent que les attaques prennent de l'ampleur d'année en année dans le verger de production du HautSassandra. Toutefois, l'importance des dégâts causés varient d'une localité à l'autre. En effet, les résultats ont révélé que dans la localité d'Issia les dégâts étaient légèrement plus importants que dans les zones de Daloa et Zoukougbeu. Le suivi de la dynamique des populations d'Eulophonotus myrmeleon dans la région du HautSassandra a révélé deux périodes de fortes attaques au cours de l'année. Ces résultats corroborent ceux obtenus par N'Guessan et al. (2010), qui avaient identifié également deux périodes de pullulation de ce ravageur dans la région du Sud-Bandama en Côte d'Ivoire. Cependant, les périodes de pullulation dans les deux régions ne se chevauchent pas. En effet, N'Guessan et al. (2010) ont indiqué que dans la région du SudBandama, les périodes de fortes attaques s'étendent de Mai à Août et de Novembre à Février. Notre étude a révélé que dans la région du Haut-Sassandra, les périodes de fortes attaques s'étalent de Janvier à Avril avec un pic en Février et de Juin à Octobre avec un pic en Août. Les décalages des périodes de pullulations d'Eulophonotus myrmeleon d'une région à l'autre peuvent 
s'expliquer par les facteurs qui régulent leurs populations. En effet, plusieurs facteurs sont susceptibles d'affecter la population de ce ravageur dans les cacaoyères dans différentes régions de production. Les facteurs climatiques, particulièrement les précipitations et la température peuvent être à l'origine des variations cycliques des populations de ces insectes (Entwistle, 1972; Wegbé et al., 1995 ; N'Guessan et al., 2010). L'effet de la pluviométrie sur la dynamique des populations a déjà été observé chez d'autres insectes. En effet, les travaux de Lavabre et al. (1962 et 1963), Bruneau De Miré (1970) et Decazy (1974) sur les variations saisonnières des populations des mirides ont montré que les précipitations n'affectent pas directement les populations de mirides, mais créent des conditions environnementales favorables à leur développement. Lavabre et al. (1963) ont indiqué que la multiplication des mirides est plutôt sous la dépendance d'un changement physiologique du cacaoyer, causé par un renouveau qualitatif et quantitatif de la sève. C'est cette succulence de la plante qui favorise l'accroissement des populations des mirides. Ainsi, la principale cause du phénomène de fluctuation des populations de mirides serait constituée par les facteurs trophiques. Nos résultats ont révélé que les périodes pluvieuses sont favorables à un accroissement du nombre de trous récents d'E. myrmeleon. En 2012 et 2013 où les précipitations étaient plus importantes, on note que les niveaux d'attaques ont été plus élevés. Ceci peut justifier le rôle important de la pluviométrie qui crée des conditions favorables dans les fluctuations des populations d'E. myrmeleon. A l'image des mirides, pour Eulophonotus, le tronc succulent du cacaoyer résultant des pluies pourrait constituer une importante source de nourriture. Ceci peut expliquer l'accroissement du nombre de trous récents après ou

\section{CONCLUSION}

Les résultats de l'étude de la dynamique des populations d'Eulophonotus myrmeleon dans la région du HautSassandra en Côte d'Ivoire ont révélé deux périodes de pullulation au cours de l'année. Ces périodes s'étalent de Janvier à Avril avec un pic en Février et de Juin à Octobre avec un pic en Août. L'étude a permis également de connaître les périodes d'émergence des adultes. Ces

\section{REMERCIMENTS}

Les auteurs remercient Messieurs ATTO N'Guessan Jean, N'DRI Kouassi Frank et AMANI Vincent De Paul, pour leur participation à la collecte des données. Les auteurs expriment également leur gratitude au FIRCA qui pendant les périodes pluvieuses. Les périodes sèches et les températures élevées contribuent généralement à la déshydratation des tissus végétaux et sont à l'origine d'une baisse de population chez certains insectes. Nos résultats ont montré que la plus grande période de forte pullulation d'Eulophonotus myrmeleon coïncide avec les températures les plus basses de l'année. Cependant, Stacey (2003) et Newman $(2005,2006)$, ont montré que l'augmentation des températures pourraient favoriser l'accroissement des populations de certains insectes phytophages. Les différences entre les périodes de fortes attaques d'E. myrmeleon observées entre deux régions peuvent s'expliquer en partie par les variations des facteurs climatiques d'une région à l'autre. Nos résultats corroborent ceux de Wegbé et al. (1995) qui ont déterminé deux périodes de fortes attaques d'E. myrmeleon dans la région du Kloto au Togo. Les deux périodes observées par Wegbé et al. (1995), de Février à Mai et Juillet à Septembre chevauchent avec celles obtenues dans notre étude. Ces similitudes pourraient s'expliquer par l'intensité des précipitations et l'effet de la température. Outre les facteurs climatiques, d'autres facteurs, notamment la présence d'ennemis naturels peuvent être à l'origine des fluctuations cycliques des populations de plusieurs insectes. Ces ennemis naturels peuvent varier d'une région à l'autre, car influencés euxmêmes par les conditions climatiques. Selon Vos et al. (2003), l'hyménoptère Glyptomorpha parasite les larves d'Eulophonotus myrmeleon. Aussi, après l'éclosion, lorsque que la larve de Eulophonotus myrmeleon commence à creuser la galerie dans le tronc du cacaoyer, elle est exposée à plusieurs ennemis naturels, notamment les fourmis et d'autres Hyménoptères (Entwistle, 1972).

résultats peuvent être utiles pour les prises de décision au regard des interventions phytosanitaires contre les larves et les adultes de ce déprédateur. En effet, les périodes de fortes attaques et les périodes d'émergence des adultes pourraient être prises en compte pour toute application rationnelle d'insecticide.

a financé les travaux pour le compte de la filière café cacao en Côte d'Ivoire. Cet article est publié avec la permission du Directeur Général du Centre National de Recherche Agronomique. 
REFERENCES

Anikwe JC, 2010. The Seasonal Occurrence and Control of the Cocoa Stem Borer, Eulophonotus myrmeleon Fldr. (Lepidoptera: Cossidae) on Cocoa in Ibadan, Nigeria. Libyan Agriculture Research Center Journal International, 1 (3): 142-146.

Bruneau De Miré P, 1970. Observations sur les fluctuations saisonnières d'une population de Sahlbergella singularis au Cameroun. Café, Cacao, Thé; 14 (3): 202-208.

Caplong P, Paulin D, Alauzet C, Decazy B, 1993. Le foreur du tronc du cacaoyer, Eulophonotus myrmeleon Felder (Lepidoptera: Cossidae), un ravageur potentiellement dangereux en cacao culture. In: Proceedings of the $11^{\text {th }}$ International Cocoa Research Conference. Yamoussoukro, Côte d'Ivoire. Cocoa Producers Alliance. Lagos, Nigeria. pp 215-221.

Daramola AM, 1978. Insect pests of kola in Nigeria. Research Bulletin No. 3 CRIN, Ibadan. 33p.

Decazy B, 1974. Les variations saisonnières des populations de Boxiopsis madagascariensis Lavabre, mirides du cacaoyer à Madagascar (Note préliminaire). Café, Cacao, Thé; 18(4): 255-262.

Entwistle P.F., 1972. Pest of cocoa. Longman Group Limited, London; 779p.

ICCO, 2008. Growing cocoa. Disponible auprès de I'organisation internationale du cacao (ICCO), www.icco.org/about/growing.aspx, consulté le 3 juillet 2008.

ICCO, 2012. L'économie cacaoyère. Ed. OPEN MIND, Le journal de L'Economie, Bulletin trimestriel de statistiques cacaoyères, volume XXXVVIII, $\mathrm{N}^{\circ} 4$, 100p.

Lavabre EM, Decelle J, Debord J, 1962. Recherches sur les variations de population de mirides (Capsides) en Côte d'Ivoire. Café, Cacao, Thé; 6(4): 287-295.

Lavabre EM, Decelle J, Debord J, 1963. Etude de l'évolution régionale et saisonnière des populations de mirides (Capsides) en Côte d'Ivoire. Café, Cacao, Thé; 7 (3): 267-289.

Ndubuaku TCN, 1989. Economic insect pests of kola. In: Progress in Tree Crop Research, 2nd edition, CRIN, Ibadan, Nigeria. pp: 115-126.

Newman JA, 2005. Climate change and the fate of cereal aphids in Southern Britain. Glob. Change Biol. 11: 940-944.

Newman JA, 2006. Using the output from global circulation models to predict changes in the distribution and abundance of cereal aphids in Canada: a mechanistic modeling approach. Glob. Change Biol. 12: 1634-1642.

N'Guessan KF, 2007. The cocoa stem borer, Eulophonotus myrmeleon Felder (Lepidoptera: Cossidae) and others new potentially dangerous Lepidopteran pests of cocoa". Proceedings of the 15th International Cocoa Research Conference, San José, Costa Rica. 9 au 14 Octobre 2006. pp 10791086.

N'Guessan KF, Kébé IB, Adiko A, 2010. Seasonal variations of the population of Eulophonotus myrmeleon Felder (Lepidoptera: Cossidae) in the Sud-Bandama region of Côte d'Ivoire. Journal of Applied Biosciences, 35: 2251-2259.

Ojo AA, 1981. Investigations into the control of the kola stem borer, Phosphorus virescens Oliver (Cerambycidae: Coleoptera) in Western Nigeria. Turrialba, 31(3): 267-270.

Okelana FA, 1989. Observations on insect pests infestation in rehabilitated plots. CRIN Ann. Report, Ibadan, Nigeria, pp 27-28.

Padi B. and Adu-Acheampong R, 2000. Preliminary measure for the control of the cocoa stem borer Eulophonotus myrmeleon Felder (Lepidoptera : Cossidae). In: Proceedings of the 13th International Cocoa Research Conference Vol. 1, Kota Kinabalu, Sabah, Malaysia, Cocoa Producers Alliance. Lagos, Nigeria. pp 649-657.

Pallix G. et Comolet A, 1996. L'impact environnemental des pratiques macro-économiques d'ajustement structurel en Côte d'Ivoire. Rapport pour la Banque. Mondiale et le Ministère Français de la Coopération, Nairobi, Kenya, 377 p.

Stacey D, 2003. Climate and biological control in organic crops. Int.J. Pest Manage. 49: 205-214.

Vos JGM, Ritchie BJ, Flood J, 2003. A la découverte du cacao. Un guide stimulant pour la formation des facilitateurs. $\mathrm{CABI}$ Biosciences, A division of $\mathrm{CAB}$ international, $115 \mathrm{p}$.

Wegbe K, Gnakpenou D, Djekpor EK, Homa S, 1995. Résultats préliminaires sur l'étude de Eulophonotus myrmeleon Felder (Lepidoptera: Cossidae), foreur du tronc du cacao: description des dégâts, dispersion, évolution des populations dans le Kloto, sud du Togo. In: Proceedings of INCOPED 1rst International Seminar. Owusu, G.K., Padi, B, Ollennu, L.A. and Owusu-manu E. (Eds.). Accra, Ghana, pp 15-22. 